\title{
Large-scale anisotropy in stably stratified rotating flows
}

\author{
R. Marino, ${ }^{1,2,3}$ P. D. Mininni, ${ }^{4}$ D. L. Rosenberg, ${ }^{5}$ and A. Pouquet ${ }^{6,1}$ \\ ${ }^{1}$ National Center for Atmospheric Research, P. O. Box 3000, Boulder, Colorado 80307, USA \\ ${ }^{2}$ Institute for Chemical-Physical Processes, Rende (CS), 87036, Italy \\ ${ }^{3}$ Space Sciences Laboratory, University of California, Berkeley, California 94720, USA \\ ${ }^{4}$ Departamento de Física, Facultad de Ciencias Exactas y Naturales, Universidad de Buenos Aires and IFIBA, \\ CONICET, Ciudad Universitaria, 1428 Buenos Aires, Argentina \\ ${ }^{5}$ National Center for Computational Sciences, Oak Ridge National Laboratory, P. O. Box 2008, Oak Ridge, Tennessee 37831, USA \\ ${ }^{6}$ Laboratory for Atmospheric and Space Physics, University of Colorado at Boulder, Boulder, Colorado 80309, USA
}

(Received 18 January 2014; revised manuscript received 27 June 2014; published 28 August 2014)

\begin{abstract}
We present results from direct numerical simulations of the Boussinesq equations in the presence of rotation and/or stratification, both in the vertical direction. The runs are forced isotropically and randomly at small scales and have spatial resolutions of up to $1024^{3}$ grid points and Reynolds numbers of $\approx 1000$. We first show that solutions with negative energy flux and inverse cascades develop in rotating turbulence, whether or not stratification is present. However, the purely stratified case is characterized instead by an early-time, highly anisotropic transfer to large scales with almost zero net isotropic energy flux. This is consistent with previous studies that observed the development of vertically sheared horizontal winds, although only at substantially later times. However, and unlike previous works, when sufficient scale separation is allowed between the forcing scale and the domain size, the kinetic energy displays a perpendicular (horizontal) spectrum with power-law behavior compatible with $\sim k_{\perp}^{-5 / 3}$, including in the absence of rotation. In this latter purely stratified case, such a spectrum is the result of a direct cascade of the energy contained in the large-scale horizontal wind, as is evidenced by a strong positive flux of energy in the parallel direction at all scales including the largest resolved scales.
\end{abstract}

DOI: 10.1103/PhysRevE.90.023018

PACS number(s): 47.55.Hd, 47.32.Ef, 47.27.ek

\section{INTRODUCTION}

The atmosphere and the global ocean are both forced by solar radiation and tides, together with surface winds and bathymetry for the ocean. The forcing scale can vary from planetary scales $\left(\approx 10^{4} \mathrm{~km}\right)$ all the way down to $1 \mathrm{~km}$ for ocean floor topography. Under large-scale quasigeostrophic balance (a balance established among gravity, rotation, and pressure gradients), the motions are quasi-two-dimensional and the flow of energy is thought to be predominantly to the large scales. However, this poses several problems, one of which is the way energy in the whole system is being transferred to and dissipated at small scales of the order of millimeters (see, e.g., Ref. [1] for a recent review, and references therein). This issue is important for the understanding of the large-scale spectrum of the atmosphere and in the closing of the global overturning circulation of the ocean, as described recently, for example, in Refs. [2,3]. With a rotation rate at midlatitudes of $\Omega \approx 10^{-4} \mathrm{~s}^{-1}$ and Brunt-Väisälä frequencies varying from $10^{-3} \mathrm{~s}^{-1}$ as in the abyssal Southern Ocean [4] to 10 times that in the atmosphere and stratosphere, there are a variety of high-Reynolds-number turbulent regimes that occur in geophysical flows. Therefore, mesoscales in the atmosphere are dominated by stratification, while the abyssal Southern Ocean has stratification and rotation of comparable magnitude (although stratification is still dominant). Of course, current supercomputers do not allow us to study realistic values of the Reynolds number for such flows, but it should be high enough to include the effect of small-scale turbulent eddies on large-scale dynamics [5], hence the need for large numerical resolution.

As a result of this variety of regimes and of the limitations in computer power, in recent years progress has been made to understand rotating stratified flows thanks to a combination of tools, including, e.g., wave turbulence approaches [6-9], reduced equations based on asymptotic expansions in a small parameter [10-13], experiments [1418], observations in the atmosphere and the ocean $[19,20]$, and direct numerical simulations [5,21-24] (see also the reviews in Refs. $[25,26])$. However, some fundamental issues have not been clarified yet. For example: What is the distribution of energy among modes (the Fourier spectrum)? What is the role of anisotropy, and under what conditions does the system self-organize and develop large-scale structures?

In many turbulent flows this self-organization takes place through a so-called inverse cascade. In particular, in a forced flow and under certain conditions, when the forcing is applied at a rather small scale compared to the size of the domain, scales larger than the forcing can be excited in a selfsimilar process. When the process results in a power-law spectrum at small wave numbers, with constant negative flux of some quantity that is conserved in the ideal limit, the system is said to sustain an inverse cascade of that quantity. It is a well-known feature of nonlinear dynamics that has been reviewed extensively [27-29], in particular in the context of two-dimensional (2D) Navier-Stokes fluids [30], and that has also been observed in astrophysical plasmas [31-33].

Direct numerical simulations have shown that rotating flows in finite domains can develop inverse cascades of energy (see, e.g., Refs. [34,35]), and recent experiments have observed the same phenomena [36]. In the case of stratified flows, the situation is less clear. Conclusions from simulations are sometimes contradictory and depend on whether the flow also rotates or not. When rotation and stratification are comparable, inverse 
cascades have been observed [37-40]. In this case, helicity is also created in the flow $[41,42]$ and can be responsible for the so-called anisotropic kinetic $\alpha$ effect instability [43], as well as for other large-scale instabilities $[44,45]$. For stratified flows with little or no rotation, it was noted in Ref. [46] that for very long times an inverse transfer develops but with no clear evidence of a self-similar spectrum at large scales. This inverse transfer generates vertically sheared horizontal winds, which were also reported in Ref. [47]. However, statistical mechanics arguments used in Refs. [48-50], based on the conservation of energy and linearized potential vorticity, lead one to argue against the existence of an inverse cascade in purely stratified flows.

Recently we showed that in the opposite limit, when stratification is weak compared with rotation, the inverse cascade that develops is faster than in the purely rotating case [51]. We have also provided evidence of the generation of a dual energy cascade in Boussinesq flows with rotation, when forcing is applied at intermediate scales [40]. In these previous studies, our investigations of the purely stratified case were restricted to the study of the isotropic Fourier modes and did not show any occurrence of an inverse energy cascade.

By means of more detailed field decompositions, we present here results from high-resolution numerical simulations of rotating and/or stratified flows (up to $1024^{3}$ spatial grid points), forced at small scales, to point out the existence of an inverse energy transfer even in Boussinesq flows without rotation. However, this transfer is highly anisotropic and differs from the fluxes in inverse cascades reported in the rotating and stratified cases. In particular, we show that while solutions with negative energy flux and inverse cascades develop in rotating stratified flows, as observed in Refs. [40,51], the purely stratified case is characterized instead by a highly anisotropic transfer with almost zero isotropic energy flux at large scales (i.e., no inverse cascade develops). In this latter case, the perpendicular kinetic energy spectrum develops a peak at scales larger than the forcing scales, which is consistent with findings in previous works that reported the development of vertically sheared horizontal winds [46,47]. However, and unlike previous works, when sufficient scale separation is allowed between the forcing scale and the domain size, the energy displays a perpendicular spectrum with power-law behavior compatible with $\sim k_{\perp}^{-5 / 3}$. This spectrum is the result of a direct cascade of the energy contained in the large-scale horizontal velocity and is accompanied by a positive flux of energy in the parallel direction at the largest scales. This result is interesting in the light of observations of a spectrum with similar scaling and with positive flux at intermediate scales in the atmosphere [52-54], as it may provide another mechanism to generate such a power law (see Refs. [23,55-59] for other explanations).

\section{SIMULATIONS OF ROTATING AND STRATIFIED FLOWS}

We use a parallelized pseudospectral code $[60,61]$ to solve the Boussinesq equations for an incompressible and
TABLE I. Parameters used in the simulations: $n_{p}$ is the linear grid resolution, $k_{F}$ the forcing wave number, Ro the Rossby number, Fr the Froude number, $k_{Z}$ the Zeman scale, and $k_{O}$ the Ozmidov scale (see text for definitions). The Reynolds number in all the runs is $\approx 1000$.

\begin{tabular}{lcccccc}
\hline \hline Run & $n_{p}$ & $k_{F}$ & Ro & Fr & $k_{Z}$ & $k_{O}$ \\
\hline I & 1024 & {$[40,41]$} & 0.08 & 0.04 & 440 & 880 \\
II & 1024 & {$[40,41]$} & $\infty$ & 0.04 & - & 512 \\
III & 512 & {$[22,23]$} & 0.08 & 0.04 & 210 & 420 \\
IV & 512 & {$[22,23]$} & $\infty$ & 0.04 & - & 250 \\
V & 512 & {$[22,23]$} & $\infty$ & 0.02 & - & 490 \\
VI & 512 & {$[22,23]$} & $\infty$ & 0.08 & - & 120 \\
VII & 512 & {$[22,23]$} & $\infty$ & 0.12 & - & 80 \\
VIII & 512 & {$[22,23]$} & 0.08 & $\infty$ & 240 & - \\
\hline \hline
\end{tabular}

stratified fluid,

$$
\begin{aligned}
\frac{\partial \mathbf{u}}{\partial t}+\mathbf{u} \cdot \nabla \mathbf{u}-\nu \nabla^{2} \mathbf{u} & =-\nabla p-N \theta \hat{z}-f \hat{z} \times \mathbf{u}+\mathbf{F}, \\
\frac{\partial \theta}{\partial t}+\mathbf{u} \cdot \nabla \theta-\kappa \nabla^{2} \theta & =N \mathbf{u} \cdot \hat{z}, \\
\nabla \cdot \mathbf{u} & =0 .
\end{aligned}
$$

Here, $\mathbf{u}$ is the velocity, $\theta$ the (potential) temperature fluctuations, and $p$ the pressure normalized by a unit mass density. The pressure is obtained self-consistently from the incompressibility condition. We consider a Prandtl number $\operatorname{Pr}=\nu / \kappa=1$, with $\nu$ the kinematic viscosity and $\kappa$ the temperature diffusivity. In Eqs. (1) and (2), $N$ is the BruntVäisälä frequency, and $f=2 \Omega$ with $\Omega$ the rotation frequency. The external mechanical force $\mathbf{F}$ is isotropic and generated randomly, applied in a shell of modes with wave numbers $k_{F}$ (resulting in a forcing length scale $L_{F}=2 \pi / \min \left\{k_{F}\right\}$ ). Although many studies of purely stratified flows use $2 \mathrm{D}$ forcing to mimic atmospheric conditions, we use isotropic three-dimensional (3D) forcing instead as we will compare simulations with pure rotation $(N=0)$ with simulations with pure stratification $(f=0)$, and we want to let the system develop in each case the level of anisotropy that is selfconsistent with the level of rotation and stratification that is externally imposed (see below). The equations are solved in a triple periodic domain of length $2 \pi$ with $n_{p}$ grid points in each direction and evolved in time using a second-order Runge-Kutta method.

The parameters of all the simulations are given in Table I. The simulations are characterized by dimensionless numbers based on a characteristic unit velocity $U=1$ : the Reynolds number

$$
\operatorname{Re}=\frac{U L_{F}}{v}
$$

the Froude number

$$
\mathrm{Fr}=\frac{U}{N L_{F}},
$$

and the Rossby number

$$
\mathrm{Ro}=\frac{U}{f L_{F}} .
$$


Time in the simulations below is measured in units of the turnover time at the forcing scale, defined as $\tau_{N L}=L_{F} / U$.

One can also define several characteristic length scales for these flows, such as the Zeman and Ozmidov scale at which isotropy recovers (when respectively under the sole influence of rotation or stratification),

$$
\ell_{Z}=2 \pi\left(\epsilon / f^{3}\right)^{1 / 2}, \quad \ell_{O}=2 \pi\left(\epsilon / N^{3}\right)^{1 / 2} .
$$

Here $\ell_{Z}$ is the Zeman scale, $\ell_{O}$ the Ozmidov scale, and $\epsilon$ the rate of energy dissipation. The wave numbers associated with these scales, respectively, $k_{Z}$ and $k_{O}$ (with $k=2 \pi / \ell$ ), are given in Table I for the different runs. Under the hypothesis that isotropy has recovered at small scale, one can also define the Kolmogorov dissipation scale,

$$
\eta=2 \pi\left(\epsilon / v^{3}\right)^{-1 / 4},
$$

with associated wave number $k_{\eta}=2 \pi / \eta$. In the $1024^{3}$ runs this wave number is $\approx 340$, while in the $512^{3}$ runs this wave number is $\approx 200$. Note that, based on these wave numbers, most of the simulations presented here are anisotropic at all scales (i.e., even at the smallest resolved scales), and there is a reasonable scale separation between the different relevant physical scales in the system.

We start the simulations from isotropic random initial conditions with a steep spectrum $\left(\sim k^{-4}\right.$ followed by an exponential decay) from $k_{F}$ to the maximum wave number $k_{\max }=n_{p} / 3$. At $t=0$, no energy is present in any Fourier mode with $k<k_{F}$. The forcing is applied during the entire run, and the systems are evolved for at least 35 turnover times $\tau_{N L}$. During the run we monitor the evolution of the total energy to see whether there is a growth that may indicate the development of an inverse cascade or the growth of large-scale structures. In practice, energy grows or decreases first during a short transient (less than $10 \tau_{N L}$ ) and then the systems evolve towards a quasistationary state with approximately constant energy or with energy growing monotonically in time when an inverse cascade develops. To verify whether the small scales in the system have reached a turbulent steady state, we monitor as well the time evolution of the enstrophy (proportional to the energy dissipation rate).

In the absence of stratification, the inertial wave frequency is $\omega_{\mathbf{k}}=f k_{\|} / k$, with $k=|\mathbf{k}|$ and with $k_{\|}$referring to the direction of imposed rotation and/or stratification and $k_{\perp}$ denoting the plane perpendicular to this. In the purely stratified case, gravity waves satisfy $\omega_{\mathbf{k}}=N k_{\perp} / k$ with $k_{\perp}=\left(k_{x}^{2}+k_{y}^{2}\right)^{1 / 2}$, and in the general case

$$
\omega_{\mathbf{k}}=k^{-1} \sqrt{N^{2} k_{\perp}^{2}+f^{2} k_{\|}^{2}} .
$$

Note that the slow modes (modes with zero frequency) in the purely rotating case are the modes with $k_{\|}=0$ (i.e., $2 \mathrm{D}$ modes), while in the purely stratified case, they are the modes with $k_{\perp}=0$. The slow zero eigenvalue modes in the general (rotating and stratified) case in a periodic box with no mean flow have a more complex expression, see, e.g., Refs. [37,50]. This further explains why we use 3D forcing, as using 2D forcing would favor direct injection of energy only in the slow modes in the purely rotating case and in a combination of slow and fast (wave) modes in any other case.
In order to characterize the anisotropy that develops in the flow, we use different definitions for the energy spectrum, as well as for anisotropic fluxes. We start from the axisymmetric kinetic energy spectrum defined as [62,63]

$$
\begin{aligned}
e\left(k_{\perp}, k_{\|}\right) & =\frac{1}{2} \sum_{\substack{k_{\perp} \leq|\mathbf{k} \times \hat{\mathbf{x}}|<k_{\perp}+1 \\
k_{\|} \leqslant \mathbf{k}<\mathbf{z}<k_{\|}+1}}|\tilde{\mathbf{u}}(\mathbf{k})|^{2} \\
& =\frac{1}{2} \int|\tilde{\mathbf{u}}(\mathbf{k})|^{2} k \sin \Theta d \phi=e(k, \Theta),
\end{aligned}
$$

with the tilde denoting Fourier transform, $\Theta=\tan ^{-1}\left(k_{\perp} / k_{\|}\right)$ the colatitude in Fourier space with respect to the vertical axis with unit vector $\hat{z}$, and $\phi$ the longitude with respect to the $x$ axis. The sum corresponds to the discrete Fourier version used in the numerical simulations, while the integral is the definition in spherical coordinates in a continuous space.

From the axisymmetric kinetic energy spectrum one can define the so-called reduced spectra $E$ as a function of $k_{\perp}, k_{\|}$, and $k$ as follows:

$$
\begin{aligned}
E\left(k_{\perp}\right) & =\int e\left(k_{\perp}, k_{\|}\right) d k_{\|}, \\
E\left(k_{\|}\right) & =\int e\left(k_{\perp}, k_{\|}\right) d k_{\perp}, \\
E(k) & =\int e(k, \Theta) k d \Theta .
\end{aligned}
$$

Note these spectra correspond to integration of the velocity correlation tensor in Fourier space respectively over cylinders, planes, and spheres. All the reduced spectra have the physical dimension of an energy density, i.e., summed over wave number they all yield, through Parseval's theorem, the kinetic energy $E_{V}=\left\langle|\mathbf{u}|^{2}\right\rangle / 2$. However, the axisymmetric spectrum $e(k, \Theta)$ has units of energy per square wave number, and, as a result, an isotropic flow with spectrum $E(k) \sim k^{-\alpha}$ has axisymmetric spectrum $e(k, \Theta) \sim k^{-\alpha-1}$. Following the same procedure, spectra for the potential energy $E_{P}$ and for the total energy $E_{T}=E_{V}+E_{P}$ can be defined.

All these spectra give information on the anisotropy of the flow in Fourier space, but they do not provide information on how energy is transferred in that space. To study the spectral transfer, we thus also consider anisotropic fluxes. We start from an axisymmetric transfer function for the total energy as follows:

$$
\begin{aligned}
t\left(k_{\perp}, k_{\|}\right)= & \int\left[\tilde{\mathbf{u}}(\mathbf{k}) \cdot(\widehat{(\mathbf{u} \cdot \nabla \mathbf{u}})_{\mathbf{k}}^{*}+\tilde{\theta}(\mathbf{k})(\widehat{\mathbf{u} \cdot \nabla \theta})_{\mathbf{k}}^{*}\right] \\
& \times k \sin \Theta d \phi+\text { c.c. }
\end{aligned}
$$

with * and c.c. denoting complex conjugate. This axisymmetric transfer function can be written in spherical coordinates as well and will be denoted as $t(k, \Theta)$.

Following the same procedure as with the energy, we can integrate over cylinders, planes, and spheres in Fourier space 
to obtain

$$
\begin{aligned}
T\left(k_{\perp}\right) & =\int t\left(k_{\perp}, k_{\|}\right) d k_{\|}, \\
T\left(k_{\|}\right) & =\int t\left(k_{\perp}, k_{\|}\right) d k_{\perp}, \\
T(k) & =\int t(k, \Theta) k d \Theta .
\end{aligned}
$$

We can finally define perpendicular, parallel, and isotropic total energy fluxes respectively as

$$
\begin{gathered}
\Pi_{T}\left(k_{\perp}\right)=-\int_{0}^{k_{\perp}} T\left(k_{\perp}^{\prime}\right) d k_{\perp}^{\prime}, \\
\Pi_{T}\left(k_{\|}\right)=-\int_{0}^{k_{\|}} T\left(k_{\|}^{\prime}\right) d k_{\|}^{\prime}, \\
\Pi_{T}(k)=-\int_{0}^{k} T\left(k^{\prime}\right) d k .
\end{gathered}
$$

These fluxes represent the total energy that goes across a cylinder, a plane, or a sphere in Fourier space per unit of time and should not be interpreted as partial fluxes (i.e., the three fluxes satisfy the condition that when integrated over all wave numbers, they are equal to zero, as necessary to satisfy total energy conservation); for more details on anisotropic fluxes, see Ref. [64].

\section{RESULTS}

\section{A. Axisymmetric spectrum}

We start with a comparison of two configurations, one for a rotating and stratified flow and the other one for a purely stratified flow. Figure 1 shows the axisymmetric kinetic energy spectrum $e\left(k_{\perp}, k_{\|}\right)$in two $1024^{3}$ simulations: the first with $N / f=2$ (i.e., rotating and stratified) and the second with $f=0$ (only stratified). The spectra are averaged in time over the range $20<t / \tau_{N L}<30$ and correspond to runs I and II in Table I. No significant differences are observed when instantaneous axisymmetric spectra (i.e., without averaging in time) are considered. The modes that are forced are visible as dark isotropic contour levels (circles) with radius $k_{F} \approx 40$, and the flows are anisotropic at all other scales as is indicated by the shape of the remaining contours. Most of the energy concentrates near the axis $k_{\perp}=0$ in the purely stratified case and close to the line $k_{\|}=2 k_{\perp}$ in the run with $N / f=2$. A run with pure rotation has most of the energy near the axis with $k_{\|}=0$ (not shown). For small scales (i.e., for wave numbers $k>k_{F}$ ) the development of these anisotropies is well known (see, e.g., Refs. [6,65]) and leads to the formation of layers and pancakelike structures in the stratified case and to the formation of columnar structures in the rotating case. In the rotating and stratified case, the slope of the line that concentrates most of the energy in spectral space (in this case, $k_{\|}=2 k_{\perp}$ ) is associated with the development of layers in real space that are tilted with respect to the horizontal plane and also with the growth of the vertical integral scale of the flow as rotation is increased [49]. The process underlying the formation of these structures in the general case is an anisotropic energy transfer resulting from resonant triads that tends to move energy towards modes with zero frequency [66],
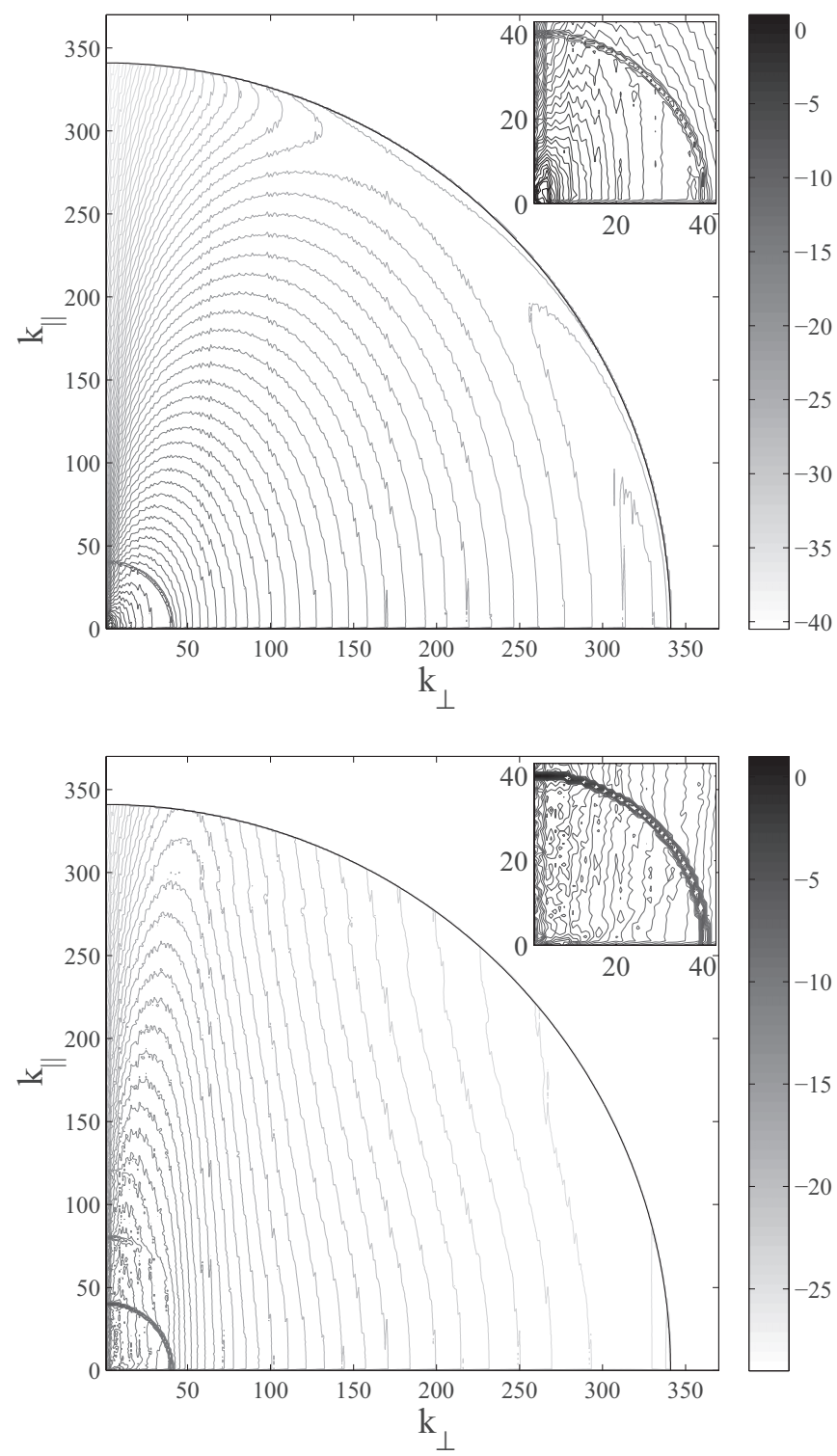

FIG. 1. Contour plots of the logarithm of the axisymmetric kinetic energy spectrum $e\left(k_{\perp}, k_{\|}\right)$for a rotating and stratified flow with $N / f=2$ (above, run I) and for a purely stratified flow with $\mathrm{Fr}=0.04$ (below, run II), averaged in time over $20<t / \tau_{N L}<30$. Note that the spherical shell of externally forced modes is clearly visible at $k \in[40,41]$. The two insets show details of the axisymmetric spectra at wave numbers smaller than or equal to the forcing wave numbers.

although strict wave resonance (without broadening) does not occur for $1 / 2 \leqslant N / f \leqslant 2$ as shown in Ref. [46] (see also Ref. [51]).

Our focus here is not on small-scale anisotropy but rather on the anisotropy that develops at scales larger than the forcing. Note that for $k<k_{F}$ the spectra are still anisotropic, and the angular distribution resembles what happens at $k>k_{F}$. In the purely stratified case, most of the energy is in modes with $k_{\perp} \approx 0$ (although, as will be shown later, energy is not concentrated in the modes with the smallest available perpendicular wave number). In the run with $N / f=2$, energy 


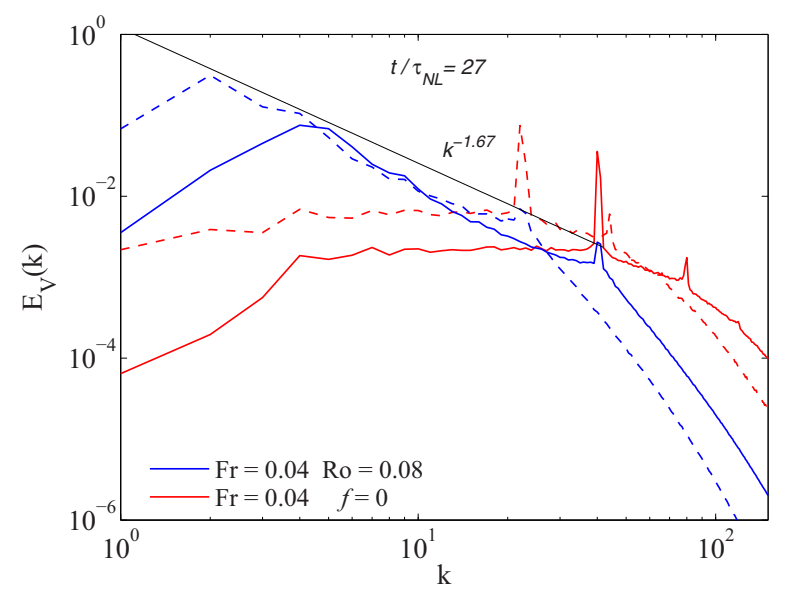

FIG. 2. (Color online) Isotropic kinetic energy spectrum $E_{V}(k)$ at $t=27 \tau_{N L}$ in simulations with the same Reynolds number, with $n_{p}=$ 512 linear grid points and forced at $k_{F}=[22,23]$ (dashed) and with $n_{p}=1024$ and $k_{F}=[40,41]$ (solid). Two cases are shown: a purely stratified case with $\mathrm{Fr}=0.04$ (light or red curves) and a rotating stratified case with $N / f=2$ (dark or blue curves). A Kolmogorov slope is indicated as a reference.

is more concentrated near the smallest wave number (i.e., at the largest scales available in the system), and, unlike the purely stratified case, the modes with small wave number have more energy than the forced wave numbers.

\section{B. Reduced spectra}

The difference in the energy contained at large scales in these systems can be also observed in the isotropic instantaneous spectra at late times, once the inverse cascade for $N / f=2$ is well developed. These spectra are shown in Fig. 2 for the two $1024^{3}$ simulations forced at $k_{F} \in[40,41]$ and also for two $512^{3}$ simulations forced at $k_{F} \in[22,23]$. While the rotating stratified flows develop a power-law spectrum at large scales (indicative of an inverse cascade), the purely stratified flows have a flat energy spectrum for $k<k_{F}$. In the purely stratified case, for $k>k_{F}$, the secondary peaks in the spectrum at $k \approx 45$ and $k \approx 81$ are likely due to harmonics of the forcing (centered at $k_{F} \approx 22.5$ in the $512^{3}$ run and at $k_{F} \approx 40.5$ for the $1024^{3}$ run). The stronger prominence of harmonic frequencies in the purely stratified case may be due to the facts that $3 \mathrm{D}$ forcing excites stronger gravity waves and that at these moderate Reynolds numbers the small-scale spectrum in the stratified case is also flatter and therefore energy in small scales is more prominent [23], a fact attributed to the accumulation of layers in the vertical direction.

In the presence of an inverse cascade, energy grows steadily at the smallest wave numbers. Since in these simulations no friction is used to remove the energy at large scales, all the analysis is stopped before accumulation (or "condensation") of energy at the gravest mode takes place, as this can result in a change in the slope of the spectrum. As an illustration, in Fig. 3 we show the spectral energy at $k=1$ as a function of time for run I (with $N / f=2$ and $n_{p}=1024$ ). Note the growth of the kinetic energy in the gravest mode as a power law of time and also note that at $t \approx 35 \tau_{N L}$ the energy $E_{V}(k=1)$ is still

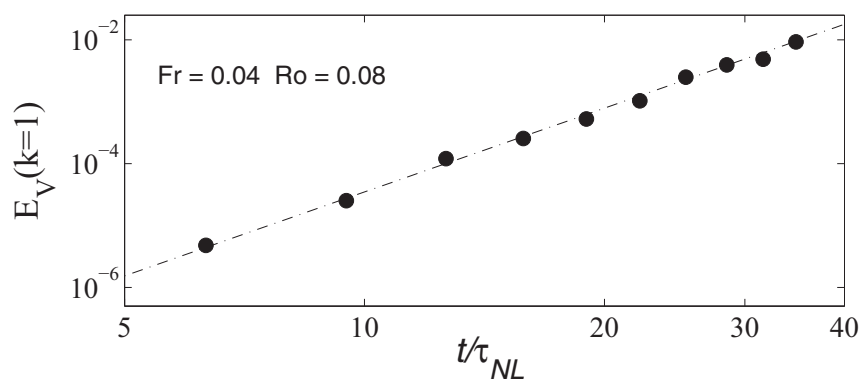

FIG. 3. Evolution of the kinetic energy in the isotropic shell in Fourier space with $k=1$ as a function of time in log-log scale for run I with $n_{p}=1024$ (see Table I). After an initial transient, the kinetic energy grows steadily with time, following a power law that is well approximated by $E_{V}(k=1, t) \sim t^{3.7}$. Accumulation of most of the energy in the $k=1$ shell does not take place in this simulation until much later times.

smaller than the energy in $k=2$ (see the solid dark curve in Fig. 2) and also smaller than the total energy in the system.

As is indicated by the axisymmetric spectrum in Fig. 1, the spectral distribution of the energy is strongly anisotropic, and the isotropic spectrum is insufficient to characterize the energy distribution at large scales. Figure 4 shows the reduced perpendicular kinetic energy spectrum $E\left(k_{\perp}\right)$ for two simulations as a function of time. In the rotating stratified flow with $N / f=2$ (run III), the energy spectrum grows at scales larger than the forcing scale, developing a power-law compatible with $\sim k_{\perp}^{-5 / 3}$ scaling. The peak of the spectrum moves towards larger scales (smaller wave numbers) as time evolves, and the energy at $k_{\perp}=1$ grows steadily in time. After $t \approx 35 \tau_{N L}$, the perpendicular kinetic energy spectrum already peaks at $k_{\perp}=1$, and the analysis is stopped. At this time, most of the kinetic energy is concentrated in the largest available perpendicular scale in the system (although not in the largest isotropic scale, as explained above).

The kinetic enstrophy $Z=\left\langle\omega^{2}\right\rangle / 2$ remains approximately constant in time after a transient, indicating the small scales in the flow have reached a steady state. A similar behavior is observed in the spectrum of the potential energy, and in the time evolution of the potential enstrophy. All these features suggest the development of an inverse cascade in the flow, which will be confirmed later by studying the energy fluxes.

The purely stratified case differs [run IV, Fig. 4(b)]. The perpendicular kinetic energy spectrum $E\left(k_{\perp}\right)$ develops rapidly a narrow range with $\sim k_{\perp}^{-5 / 3}$ between $k_{\perp} \approx 4$ and $k_{F}$ (already visible at $t=7 \tau_{N L}$, albeit in a narrower range of wave numbers). This range of scales is followed at larger scales by a flat spectrum, as observed also in the isotropic spectrum $E(k)$ in Fig. 2. Evidence of flat spectra at large scale was already provided in simulations of stably stratified turbulence with an applied 2D forcing [67] (it must be pointed out that in that case, the absence of the vertical component of the forcing does not prevent the horizontal velocity field from developing a vertical energy flux in spectral space). In run IV, even for very long times $\left(t=133 \tau_{N L}\right)$ the flat spectrum at large scales persists, and the energy at $k_{\perp}=1$ does not grow significantly. The kinetic enstrophy $Z$ remains approximately constant in time after a transient (as in the run with $N / f=2$ ), but in this 

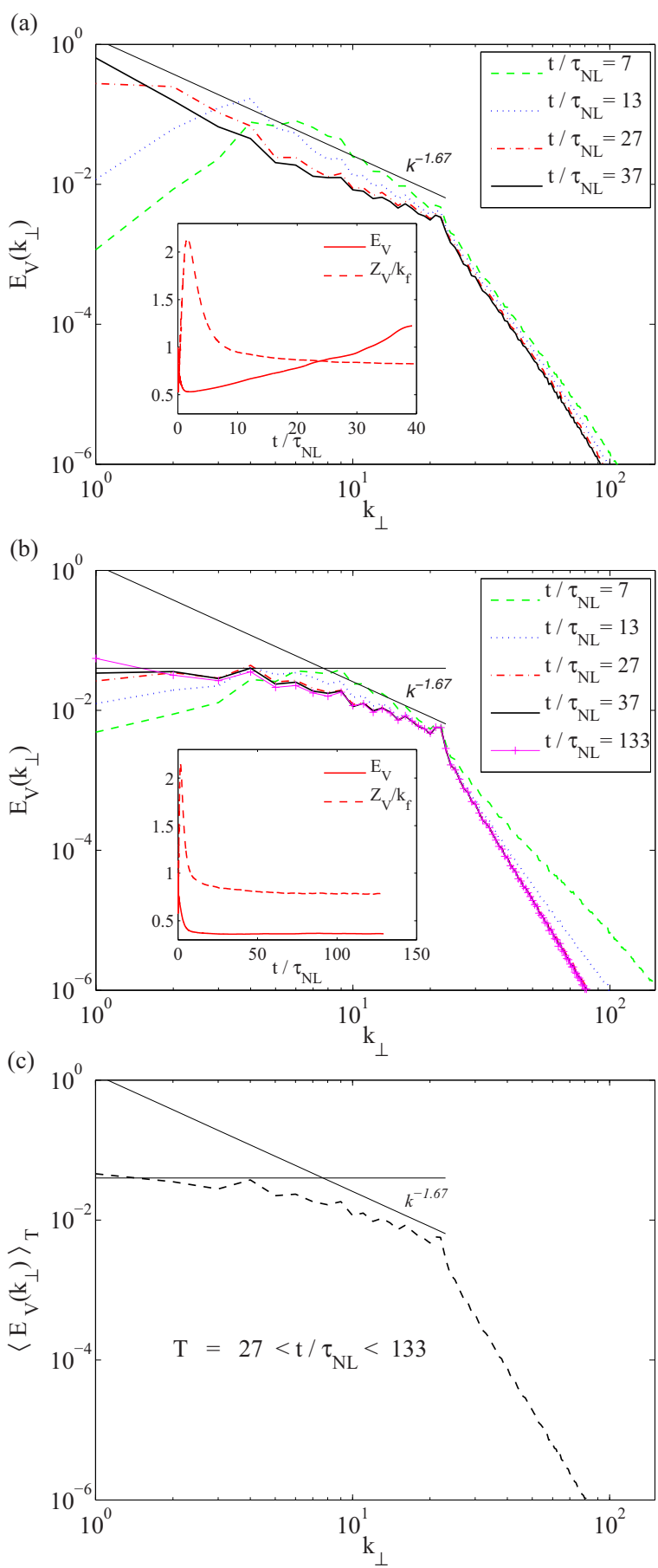

FIG. 4. (Color online) Perpendicular kinetic energy spectra $E_{V}\left(k_{\perp}\right)$ at different times for a simulation with $N / f=2$ [run III, panel (a)] and for a purely stratified flow [run IV, panel (b)], both with $\mathrm{Fr}=0.04$. A Kolmogorov slope and a flat line are indicated as references. The insets in panels (a) and (b) show the time evolution of the kinetic energy and of the (normalized) kinetic enstrophy in the same runs (solid and dashed lines respectively). Note the saturation of the kinetic enstrophy (i.e., of the power at the small scales) in both cases and of kinetic energy only in the purely stratified case. Panel (c) shows a time average over $\approx 100 \tau_{N L}$ of the the perpendicular kinetic energy spectrum for the purely stratified run of panel (b) (run IV).

case the kinetic energy also remains approximately constant (unlike the rotating and stratified case).

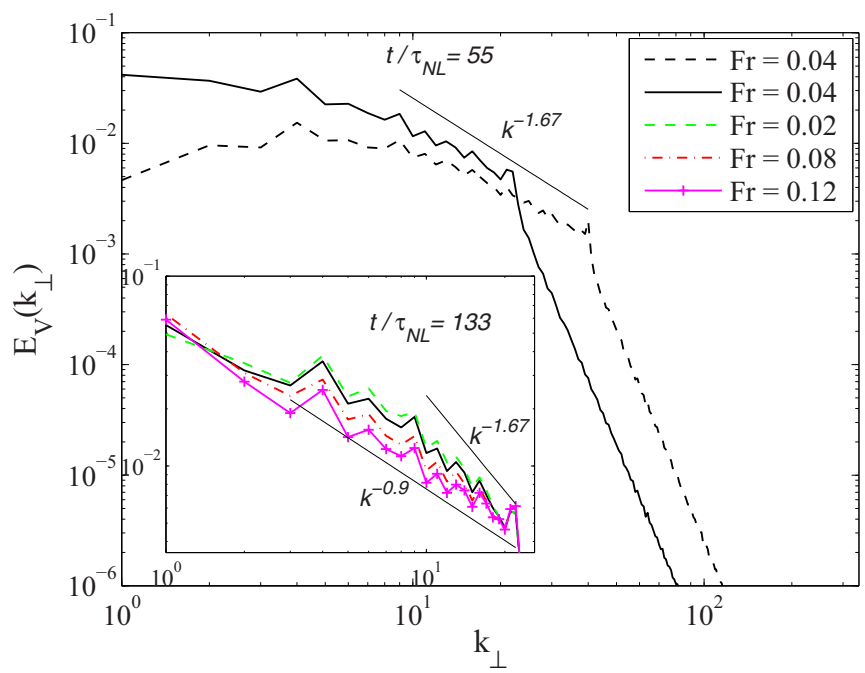

FIG. 5. (Color online) Perpendicular kinetic energy spectra $E_{V}\left(k_{\perp}\right)$ at $t=55 \tau_{N L}$ for two purely stratified flows (runs II and IV) using grids with $n_{p}=512$ (solid) and $n_{p}=1024$ (dashed) points, with the same Froude and Reynolds numbers. A Kolmogorov slope is given as a reference. The inset shows details of the spectra for small wave numbers at later times for several runs with $\mathrm{Re} \approx 1000$ (runs IV to VII) without rotation and with different Froude numbers. The labels in the top right box correspond to the curves in this inset.

In order to demonstrate the persistence of the large-scale behavior of $E\left(k_{\perp}\right)$ in the purely stratified case, we averaged the spectrum over roughly 100 turnover times, i.e., over the interval $27<t / \tau_{N L}<133$, as shown in Fig. 4(c). A transition between a flat spectrum to a steeper spectrum can still be observed at $k_{\perp} \approx 4$ in the time-averaged reduced perpendicular spectrum.

The differences in the run with $f=0$ between the isotropic and perpendicular kinetic energy spectra are associated with the fact that the axisymmetric spectrum is highly anisotropic, with most of the energy concentrated in modes close to the axis with $k_{\perp}=0$. As a result, integration of $e(k, \Theta)$ over circles or over planes with constant $k_{\perp}$ yields different reduced spectra. Is the reduced perpendicular spectrum an indication of an inverse cascade in the purely stratified case, even though it does not peak at the largest available perpendicular scale? It is interesting to point out that a spectrum peaking at intermediate wave numbers was observed before in numerical simulations [46]. However, in the simulations in Ref. [46] no power law was found between the peak at intermediate wave numbers and $k_{F}$, probably because the simulations were done at lower resolution and with less scale separation at large scales.

To find the origin of this spectrum, we first study whether the peak of $E\left(k_{\perp}\right)$ at $k \approx 4$ in the stratified case depends on the scale separation or on the Froude number. Figure 5 shows a run with $n_{p}=512$ forced at $k_{F} \in[22,23]$ (run IV) and a run with $n_{p}=1024$ forced at $k_{F} \in[40,41]$ (run II), both with $\mathrm{Fr}=0.04, \operatorname{Re} \approx 1000$, and $f=0$. This value of the Reynolds number is somewhat constrained by the choice of a small-scale forcing but is still high enough for the flow to develop a power-law turbulent spectrum. In spite of the change 

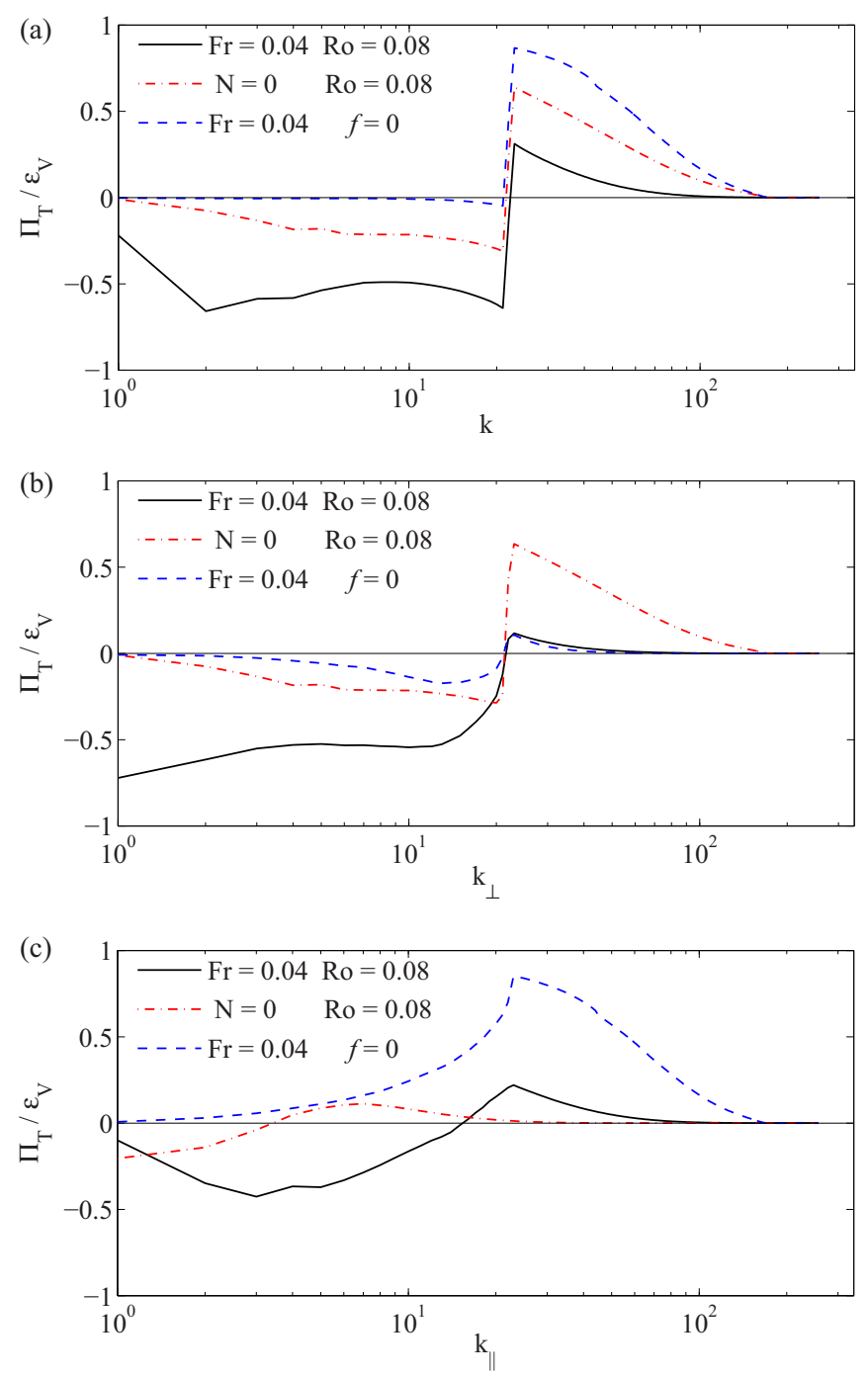

FIG. 6. (Color online) (a) Isotropic, (b) perpendicular, and (c) parallel total energy fluxes normalized by the energy input $\epsilon_{V}=$ $\langle\mathbf{u} \cdot \mathbf{F}\rangle$ in a run with $N / f=2$ (solid black line), a run with pure rotation (dash-dotted red line), and a run with pure stratification (dashed blue line).

in $k_{F}$, both runs show a peak at $k_{\perp} \approx 4$ at the same time in units of turnover time, with an approximately flat spectrum at smaller wave numbers. The inset in Fig. 5 shows multiple $n_{p}=512$ runs (runs IV to VII) all forced at $k_{F} \in[22,23]$, all with the same Reynolds number but now with different Froude numbers varying from 0.04 to 0.12 (see inset). As the effect of stratification becomes weaker (larger Froude number), the $-5 / 3$ range weakens as well and becomes shallower although, interestingly, a peak at $k_{\perp} \approx 1$ develops for the largest Fr. This could be attributed to the fact that, with a flatter spectrum, it is faster for the energy to be available to larger scales.

\section{Fluxes and energy transfer}

We consider now the total energy fluxes defined in the previous section in Eqs. (18)-(20). The fluxes are displayed in Fig. 6 as a function of isotropic, perpendicular, and parallel wave numbers for three runs with $n_{p}=512$ : one run with $\mathrm{Fr}=0.04$ and $\mathrm{Ro}=0.08$ (rotating and stratified, with $N / f=$ 2 , run III), one run with Ro $=0.08$ and $N=0$ (purely rotating, run VIII), and one run with $\mathrm{Fr}=0.04$ and $f=0$ (purely stratified, run IV). For $k>k_{F}$, the fluxes are positive in all the runs, indicating a direct cascade of total energy in the three cases. To smooth out local temporal fluctuations of the energy transfer, fluxes are averaged over the range $20<t / \tau_{N L}<30$. This choice for the time average appears to be consistent with the quasistationarity of the inverse cascade as suggested in Ref. [68].

Although the scale separation between the minimum and forcing wave numbers is insufficient to observe a range with constant fluxes, the sign of the flux is a clear indication of the direction of the energy transfer.

At scales larger than the forcing scale, or for $k<k_{F}$, the purely rotating run (dash-dotted red curves in Fig. 6) has negative $\Pi_{T}(k)$ and $\Pi_{T}\left(k_{\perp}\right)$ fluxes. Note also that for that same run, $\Pi_{T}\left(k_{\|}\right)$is smaller and changing sign, although negative values do prevail for the smallest wave numbers. Moreover, in $\Pi_{T}(k)$ and $\Pi_{T}\left(k_{\perp}\right)$, a range of wave numbers can be identified at large scales for which the fluxes remain approximately constant. This is consistent with what was observed in previous studies (see, e.g., Refs. [35,46]): In the purely rotating case, energy at large scales goes towards $2 \mathrm{D}$ modes (modes with $k_{\|}=0$ ), modes which then undergo an inverse cascade in the $2 \mathrm{D}$ plane towards the largest scales in the system.

In the rotating and stratified run with $N / f=2$ (solid black line), a similar behavior is observed, but the inverse fluxes are larger in absolute value (i.e., the inverse cascade is faster) than in the purely rotating case, while the direct fluxes are smaller. This is studied in more detail in Ref. [51]; it is associated with the fact that for $N / f=2$ there are no resonant triads [46] and energy can be transferred more efficiently towards the $2 \mathrm{D}$ modes. For larger values of $N / f$ the inverse cascade persists (see, for example, Refs. [37,38,46,51]), although the inverse cascade becomes slower (less efficient) as $N / f$ is increased.

Finally, and more importantly, we find that the purely stratified case differs from the other two cases (dashed blue line in Fig. 6). The isotropic flux $\Pi_{T}(k)$ at $k<k_{F}$ is almost zero, indicating that almost no net energy goes across spheres in Fourier space for small wave numbers. This stems from different behaviors in the parallel and perpendicular directions. Indeed, the perpendicular flux $\Pi_{T}\left(k_{\perp}\right)$ shows a range with negative values (albeit much smaller than in the rotating case or in the rotating and stratified case) and becomes negligible for $k_{\perp} \lesssim 4$. The parallel flux $\Pi_{T}\left(k_{\|}\right)$ is positive and dominant for all wave numbers, indicating a strong transfer towards smaller vertical scales, although this flux decreases rather sharply as we approach the largest scale.

Considering now scales smaller than the forcing, or for $k>k_{F}$, we note the stronger direct (positive) flux in the purely stratified case, dominated by the transfer in the vertical direction which corresponds to the slow modes of the system with $k_{\perp}=0$, whereas the transfer in the horizontal plane is stronger for pure rotation (again, for the slow mode, with now $\left.k_{\|}=0\right)$. 

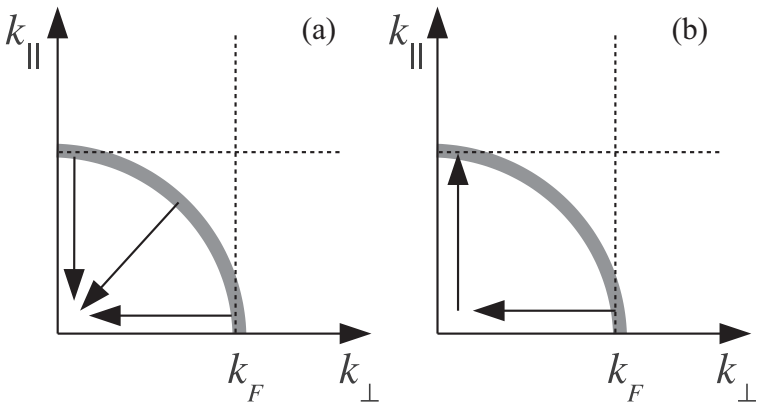

FIG. 7. Schematic representation of the total energy flux in Fourier space, defined in Eqs. (18)-(20) for (a) rotating (or rotating and stratified) flows and (b) the purely stratified case. The gray circle indicates the shell of forced modes, and the dashed lines indicate planes across which the fluxes $\Pi_{T}\left(k_{\perp}\right)$ (represented by horizontal arrows) and $\Pi_{T}\left(k_{\|}\right)$(represented by vertical arrows) are computed. The diagonal arrow represents the flux in terms of isotropic wave number, $\Pi_{T}(k)$ which, in case (b), is negligible with two projections (on the $k_{\perp}$ and $k_{\|}$directions) that are almost equal but of opposite sign. Note, however, that the length of the arrows is arbitrary and does not denote the strength of each flux.

\section{DISCUSSION AND CONCLUSION}

In Fig. 7 we present a schematic representation of these fluxes for all cases considered (rotating, rotating and stratified, and purely stratified). The isotropic flux $\Pi_{T}(k)$ measures how much energy goes per unit of time across circles in the $\left(k_{\|}, k_{\perp}\right)$ plane (spheres in 3D Fourier space); for rotating flows, as well as for rotating and stratified flows, it is negative for $k<k_{F}$. The fluxes $\Pi_{T}\left(k_{\perp}\right)$ and $\Pi_{T}\left(k_{\|}\right)$measure how much energy per unit of time crosses lines respectively with constant $k_{\perp}$ or $k_{\|}$ (i.e., cylinders or planes in $3 \mathrm{D}$ Fourier space). In all the runs with nonzero rotation, all the fluxes are negative, resulting in an accumulation of a fraction of the injected energy at the smallest available wave numbers.

The purely stratified run has, for $k<k_{F}$, almost no net energy going across circles. This is the result of a competition between an inverse flux in $\Pi_{T}\left(k_{\perp}\right)$ and a direct flux in $\Pi_{T}\left(k_{\|}\right)$. A fraction of the energy forced in the shell $k=k_{F}$ goes towards wave numbers with smaller $k_{\perp}$, larger $k_{\|}$, or strata. This process is known to result in the development of vertically sheared horizontal winds [46], and, with sufficient scale separation, as is the case in our simulations, also results in the $\sim k_{\perp}^{-5 / 3}$ spectrum observed for a range of wave numbers with $k_{\perp}<k_{F}$. However, energy is also transferred directly towards larger $k_{\|}$, as indicated by $\Pi_{T}\left(k_{\|}\right)>0$, and this prevents energy from reaching $k=1$ and results in a flat spectrum for the smallest wave numbers.

Several other forcing mechanisms have been put forward in the literature, such as 2D forcing [69,70], as well as an assembly of vortex dipoles [71]. Each deserves separate studies in view of the complexity of these flows, and of the several characteristic scales in the system, as well as the different inertial ranges that have to be resolved $a$ priori. The question as to whether universal results will ensue is still open and will require ample further studies at high resolution. We note that the aspect ratio of the fluid is also a factor to be concerned with
[69], as emphasized recently for rotating flows [70] as well as for stratified flows [72].

We therefore cannot rule out at this point a dependency of the observed fluxes on the specific kind of forcing used and on the strength of waves. Further investigations along these lines are left for a future study where the level of anisotropy in the forcing will be varied to explore its effect in the cascades.

More complex stratified and rotating flows can also be envisaged in the future, with a wealth of new phenomena occurring, taking into consideration, for example, the effect of the walls on the energy budget $[73,74]$ or the dependence of viscosity and diffusivity on temperature [75], leading in that case to intermittent bursts of heat transfer (see also Ref. [76] for strong intermittency in stratified flows). This opens the question of the validity of the assumption of a unit Prandtl number, at least in modeling approaches [75], the effect on thermal expansion seemingly being more important than that on viscosity [77].

In conclusion, we have presented several direct numerical simulations of stratified and/or rotating turbulence, with spatial resolutions up to $1024^{3}$ grid points and forced at small scales to have sufficient scale separation to study the development of inverse cascades. While purely rotating flows, and rotating and stratified flows at moderate values of $N / f$ develop inverse cascades, purely stratified flows have almost zero isotropic energy flux at large scales. However, there is a small negative perpendicular flux towards small $k_{\perp}$ that results in the development of vertically sheared horizontal winds. Unlike in previous works, the present study shows that when sufficient scale separation is allowed between the forcing scale and the domain size, the kinetic energy spectrum at large scales displays a power-law behavior in the perpendicular direction compatible with $\sim k_{\perp}^{-5 / 3}$. This spectrum is the result of the combined inverse transfer in the perpendicular direction and a direct cascade of the large-scale horizontal winds manifested by a positive flux of energy in the parallel direction at the largest scales. This highly anisotropic transfer in stratified flows can result in the buildup of a power-law spectrum at large scales (albeit up to some wave number and followed at larger scales by a flat spectrum), with positive flux in some directions in spectral space.

\section{ACKNOWLEDGMENTS}

This work was supported by NSF/CMG Grant No. 1025183; it was also sponsored by an NSF cooperative agreement through the University Corporation for Atmospheric Research on behalf of the National Center for Atmospheric Research (NCAR). Computer time was provided on NSF/XSEDE TG-PHY100029 and 110044 and NCAR/ASD on Yellowstone. P.D.M. acknowledges support from UBACYT Grant No. 20020110200359, PICT Grants No. 2011-1529 and No. 2011-1626, and PIP Grant No. 11220090100825. A.P. acknowledges support from LASP and, in particular, Bob Ergun. R.M. acknowledges the Regional Operative Program Calabria ESF 2007/2013 and the Marie Curie Project FP7 PIRSES-2010-269297 “Turboplasmas.” 
[1] E. Deusebio, A. Vallgren, and E. Lindborg, J. Fluid Mech. 720 66 (2013).

[2] A. M. Thurnherr and L. C. S. Laurent, Geophys. Res. Lett. 38, L15613 (2011).

[3] M. Nikurashin and R. Ferrari, Geophys. Res. Lett. 40, 3133 (2013).

[4] M. Nikurashin, G. K. Vallis, and A. Adcroft, Nat. Geosci. 6, 48 (2012).

[5] P. Bartello and S. M. Tobias, J. Fluid Mech. 725, 1 (2013).

[6] C. Cambon, Eur. J. Mech. B Fluids 20, 489 (2001).

[7] A. Newell, S. Nazarenko, and L. Biven, Physica D 152-153, 520 (2001).

[8] P. Sagaut and C. Cambon, Homogeneous Turbulence Dynamics (Cambridge University Press, Cambridge, 2008).

[9] S. Nazarenko, Wave Turbulence, Vol. 825 (Springer-Verlag, Berlin, 2011).

[10] P. Embid and A. Majda, Geophys. Astrophys. Fluid Dyn. 87, 1 (1998).

[11] K. Julien, E. Knobloch, R. Milliff, and J. Werne, J. Fluid Mech. 555, 233 (2006).

[12] R. Klein, Ann. Rev. Fluid Mech. 42, 249 (2010).

[13] B. A. Wingate, P. Embid, M. Holmes-Cerfon, and M. A. Taylor, J. Fluid Mech. 676, 546 (2011).

[14] C. Baroud, B. Plapp, H. Swinney, and Z. She, Phys. Fluids 15, 2091 (2003).

[15] C. D. Troy and J. R. Koseff, Exp. Fluids 38, 549 (2005).

[16] O. Praud, J. Sommeria, and A. Fincham, J. Fluid Mech. 547, 389 (2006).

[17] L. van Bokhoven, H. Clercx, G. van Heijst, and R. Trieling, Phys. Fluids 21, 096601 (2009).

[18] G. Bordes, F. Moisy, T. Dauxois, and P. Cortet, Phys. Fluids 24, 014105 (2012).

[19] G. D. Nastrom and F. D. Eaton, J. Geophys. Res. 111, D19103 (2006).

[20] Z. Li, W. Robinson, and A. Liu, J. Geophys. Res. 114, D14103 (2009).

[21] H. Aluie and S. Kurien, Eur. Phys. Lett. 96, 44006 (2011).

[22] M. Waite, Phys. Fluids 23, 066602 (2011).

[23] Y. Kimura and J. R. Herring, J. Fluid Mech. 698, 19 (2012).

[24] S. Almalkie and S. M. de Bruyn Kops, J. Turb. 13, N29 (2012).

[25] J. Riley and M.-P. Lelong, Ann. Rev. Fluid Mech. 32, 613 (2000).

[26] C. Staquet and J. Sommeria, Ann. Rev. Fluid Mech. 34, 559 (2002).

[27] L. Biferale, S. Musacchio, and F. Toschi, Phys. Rev. Lett. 108, 164501 (2012).

[28] G. Boffetta and R. E. Ecke, Ann. Rev. Fluid Mech. 44, 427 (2012).

[29] A. Pouquet, A. Sen, D. Rosenberg, P. D. Mininni, and J. Baerenzung, Phys. Scr. T155, 014032 (2013).

[30] R. Kraichnan and D. Montgomery, Rep. Prog. Phys. 43, 547 (1980).

[31] R. Marino, L. Sorriso-Valvo, V. Carbone, A. Noullez, R. Bruno, and B. Bavassano, Astrophys. J. 677, L71 (2008).

[32] R. Marino, L. Sorriso-Valvo, V. Carbone, P. Veltri, A. Noullez, and R. Bruno, Planet. Space Sci. 59, 592 (2011).

[33] R. Marino, L. Sorriso-Valvo, R. D'Amicis, V. Carbone, R. Bruno, and P. Veltri, Astrophys. J. 750, 41 (2012).
[34] L. M. Smith and F. Waleffe, Phys. Fluids 11, 1608 (1999).

[35] A. Sen, P. D. Mininni, D. Rosenberg, and A. Pouquet, Phys. Rev. E 86, 036319 (2012).

[36] E. Yarom, Y. Vardi, and E. Sharon, Phys. Fluids 25, 085105 (2013).

[37] P. Bartello, J. Atmos. Sci. 52, 4410 (1995).

[38] O. Métais, P. Bartello, E. Garniera, J. J. Riley, and M. Lesieur, Dyn. Oc. Atm. 23, 193 (1996).

[39] S. Kurien, B. Wingate, and M. Taylor, Eur. Phys. Lett. 84, 24003 (2008).

[40] A. Pouquet and R. Marino, Phys. Rev. Lett. 111, 234501 (2013).

[41] R. Hide, Geophys. Fluid Dyn. 7, 157 (1976).

[42] R. Marino, P. D. Mininni, D. Rosenberg, and A. Pouquet, Phys. Rev. E 87, 033016 (2013).

[43] U. Frisch, Z. She, and P. Sulem, Physica D 28, 382 (1987).

[44] A. Tur and V. Yanovsky, Open J. Fluid Dyn. 03, 64 (2013).

[45] A. Tur, M. Chabane, and V. Yanovsky, Open J. Fluid Dyn. 03, 340 (2013).

[46] L. M. Smith and F. Waleffe, J. Fluid Mech. 451, 145 (2002).

[47] J.-P. Laval, J. C. McWilliams, and B. Dubrulle, Phys. Rev. E 68, 036308 (2003)

[48] M. Waite and P. Bartello, J. Fluid Mech. 517, 281 (2004).

[49] M. Waite and P. Bartello, J. Fluid Mech. 568, 89 (2006).

[50] C. Herbert, A. Pouquet, and R. Marino, arXiv:1401.2103 (2014).

[51] R. Marino, P. D. Mininni, D. Rosenberg, and A. Pouquet, Eur. Phys. Lett. 102, 44006 (2013).

[52] G. Nastrom, W. Jasperson, and K. Gage, Nature 310, 36 (1984).

[53] E. Lindborg, J. Fluid Mech. 388, 259 (1999).

[54] P. Héas, E. Mémin, D. Heitz, and P. D. Mininni, Tellus A 64, 10962 (2012).

[55] K. K. Tung and W. W. Orlando, J. Atmos. Sc. 60, 824 (2003).

[56] R. Tulloch and K. S. Smith, Proc. Natl. Acad. Sci. USA 103, 14690 (2006).

[57] G. Brethouwer, P. Billant, E. Lindborg, and J. M. Chomaz, J. Fluid Mech. 585, 343 (2007).

[58] J. J. Riley and E. Lindborg, J. Atmos. Sc. 65, 2416 (2008).

[59] T. Teitelbaum and P. D. Mininni, Phys. Rev. E 86, 016323 (2012).

[60] D. Gómez, P. D. Mininni, and P. Dmitruk, Adv. Sp. Res. 35, 899 (2005).

[61] P. D. Mininni, D. Rosenberg, R. Reddy, and A. Pouquet, Parallel Comput. 37, 316 (2011).

[62] F. S. Godeferd and C. Staquet, J. Fluid Mech. 486, 115 (2003).

[63] P. D. Mininni, D. Rosenberg, and A. Pouquet, J. Fluid Mech. 699, 263 (2012).

[64] P. D. Mininni, Ann. Rev. Fluid Mech. 43, 377 (2011).

[65] L. Liechtenstein, F. S. Godeferd, and C. Cambon, J. Turbulence 6, 24 (2005).

[66] F. Waleffe, Phys. Fluids A 5, 677 (1993).

[67] J. Herring and O. Métais, J. Fluid Mech. 202, 97 (2006).

[68] R. Kraichnan, Phys. Fluids 10, 1417 (1967).

[69] L. M. Smith, J. R. Chasnov, and F. Waleffe, Phys. Rev. Lett. 77, 2467 (1996). 
[70] E. Deusebio, G. Boffetta, E. Lindborg, and S. Musacchio, arXiv:1401.5620 (2014).

[71] P. Augier, P. Billant, and J.-M. Chomaz, J. Fluid Mech. (unpublished).

[72] A. Sozza, G. Boffetta, P. Muratore-Ginanneschi, and S. Musacchio, arXiv:1405.7824 (2014).

[73] F. Zonta, C. Marchioli, and A. Soldati, J. Fluid Mech. 697, 150 (2012).
[74] F. Zonta, M. Onorato, and A. Soldati, J. Fluid Mech. 697, 175 (2012).

[75] F. Zonta, Int. J. Heat Fluid Flow 44, 489 (2013).

[76] C. Rorai, P. D. Mininni, and A. Pouquet, Phys. Rev. E 89, 043002 (2014).

[77] F. Zonta and A. Soldati, J. Heat Trans. ASME 136, 022501 (2013). 